\title{
Examining Intermediate Data Reduction Algorithms for use with t-SNE
}

\author{
Aaron Campbell ${ }^{*}$ \\ South Dakota School of Mines and \\ Technology \\ Rapid City, South Dakota \\ aaron.campbell@mines.sdsmt.edu
}

\author{
Kyle Caudle ${ }^{\dagger}$ \\ South Dakota School of Mines and \\ Technology \\ Rapid City, South Dakota \\ kyle.caudle@sdsmt.edu
}

\author{
Randy C. Hoover ${ }^{\ddagger}$ \\ South Dakota School of Mines and \\ Technology \\ Rapid City, South Dakota \\ randy.hoover@sdsmt.edu
}

\begin{abstract}
t-distributed Stochastic Neighbor Embedding (t-SNE) is a data visualization tool that was developed to provide a flexible, nonparametric method for mapping high dimensional data onto a two or three dimensional subspace for data visualization. This paper observes the effects of using different intermediate data reduction algorithms (e.g., Principal Component Analysis, Independent Component Analysis, Linear Discriminant Analysis, Sammon Mapping, and Local Linear Embedding) to first reduce the data to an intermediate subspace prior to applying t-SNE for visualization. Our research shows that no intermediate step in the visualization process is trivial, and application dependent knowledge should be utilized to ensure the best possible visualization in lower dimensional spaces. Experimental results are presented for several common data sets where we illustrate that, for clustering applications and visualization of class separation of multi-class data, each algorithm tested results in significantly different mappings.
\end{abstract}

\section{CCS CONCEPTS}

- Computing methodologies $\rightarrow$ Machine learning algorithms;

\section{KEYWORDS}

data reduction, data visualization, clustering

\section{INTRODUCTION}

In the modern world, vast amounts of data are being collected and analyzed for a multitude of application domains. "Big Data" analysis is a necessary and growing trend in nearly all fields of science, engineering, business and medical research. Researchers are often troubled by data sets that involve many records (deep) and many

${ }^{*}$ Mr. Campbell is currently an undergraduate student in the Computer Science and Applied/Computational Mathematics Program

${ }^{\dagger}$ Dr. Caudle is currently an Associate Professor of Statistics in the department of Mathematics and Computer Science.

$\ddagger$ Dr. Hoover is currently an Associate Professor of Computer Engineering in the department of Electrical and Computer Engineering.

Permission to make digital or hard copies of all or part of this work for personal or classroom use is granted without fee provided that copies are not made or distributed for profit or commercial advantage and that copies bear this notice and the full citation on the first page. Copyrights for components of this work owned by others than ACM must be honored. Abstracting with credit is permitted. To copy otherwise, or republish, to post on servers or to redistribute to lists, requires prior specific permission and/or a fee. Request permissions from permissions@acm.org.

ICCDA 2019, March 14-17, 2019, Kahului, HI, USA

(C) 2019 Association for Computing Machinery.

ACM ISBN 978-1-4503-6634-2/19/03 . \$ \$15.00

https://doi.org/10.1145/3314545.3314549 variables (wide). A first step in data analysis often involves visualization of the data. The old adage, "A picture is worth a thousands words", has never been more appropriate. Because visualization in more than 3 dimensions is difficult for most humans, a very useful first step involves reducing the dimensionality of the data, while attempting to maintain the underlying structure.

Dimensionality reduction, specifically for data visualization, has become a thriving area of research. This paper will focus on the a dimensionality reduction technique known as t-distributed stochastic neighbor embedding, or t-SNE [1], which is an extension of its predecessor Stochastic Neighbor Embedding (SNE) [2]. t-SNE and SNE are non-linear dimensionality reduction techniques that preserve the neighborhood structure of the data using probability distributions.

Since its inception, $\mathrm{t}$-SNE has been used in various applications to provide high quality visualization. In the medical field for example, $\mathrm{t}$-SNE has been used to help identify the malignancy of breast lesions by mapping a large number of features about the lesion to a much smaller feature space [3]. t-SNE has also been used to visualize the features learned by neural networks in varied musical applications [4].

With all dimensionality reduction algorithms, information loss is inevitable. Different algorithms attempt to maintain different aspects of the data which is being projected to a lower dimensional space. For example, Sammon Mapping and multi-dimensional scaling attempt to preserve the distance structure between points $[5,6]$. Principal Component Analysis (PCA) is a linear dimensionality reduction technique that projects the data into a lower dimensional subspace so that the variance retained is maximized [7]. SNE attempts to preserve the neighborhood structure of the data by imposing Gaussian distributions on the points in both the high and low-dimensional representations and iterating to find a representation that minimizes the differences in those distributions (the details of which shall be discussed in Section 2).

Since its inception, SNE has suffered from a common data reduction problem known as the crowding problem, which is not exclusive to SNE but inherent to the data reduction process. It presents itself whenever points of moderate distance are mapped from a high dimensional space to a lower dimensional space. The crowding occurs because there is less area in the lower dimensional space to accurately preserve the high dimensional structure. Figure 1 shows a very simple but clear example of this problem. Essentially, there is not enough space in lower dimensions to allow a truly accurate mapping that mirrors the higher dimension in both local 
and global respects. This problem only increases as the difference in dimensionality increases. While this phenomenon's effect cannot be resolved completely, different methods can be employed to mitigate its limitations. Toward this end, van Der Maaten and Hinton worked

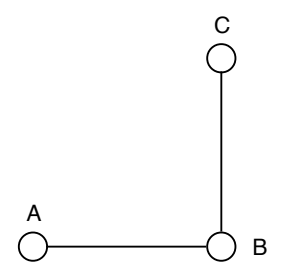

(a) High-Dimensional Representation

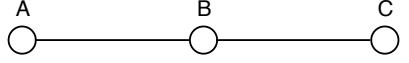

(b) Low-Dimensional Representation
Figure 1: A simple example of the Crowding Problem. If (a) is the high-dimensional space with points $A$ and $B$ being 1 unit apart, then $A$ and $C$ are $\sqrt{2}$ units apart. When these points are projected down to the low-dimensional space in (b), though, the short, local structure of A-to-B and B-to-C are maintained, but there is not enough room for A-to-C to be accurately mapped. As most reductions are more than one dimension, this problem only increases.

to develop t-SNE, an alteration to SNE with the intention of reducing the over crowding effect. The differences in the algorithm will be discussed in Section 2.

In theory, the t-SNE algorithm can be run on a data sets of any dimension. Unfortunately, the time and memory complexity of this algorithm both scale quadratically with respect to the size of the data [1]; and while it has been shown that tree-based approximations can reduce this complexity to $O(n \log n)$ [8], the use of an intermediate algorithm to first map the data into a subspace is still widely used when visualizing with t-SNE. In [1], PCA was used to reduce the dimensionality of the data to a 30-dimensional subspace prior to visualization with t-SNE. While PCA is a well-known linear dimensionality reduction technique, there are many other techniques that can be applied prior to t-SNE to form this reduced dimensional intermediate space.

The major contribution of this paper is to illustrate that using other dimensionality reduction algorithms prior to applying t-SNE can provide significantly different results and in instances where a certain trait is desirable (e.g. separating multi-class data), specific intermediate algorithms are better suited. For our experiments, both linear, non-linear, unsupervised, and supervised intermediate data reduction algorithms will be evaluated.

The remainder of this paper is organized as follows. Section 2 provides a detailed technical presentation of the t-SNE algorithm. In Section 3 we provide an overview of the intermediate dimensionality reduction techniques used in the current work. Section 4 outlines the experimental setup that will be used to compare the intermediate dimensionality reduction techniques prior to running t-SNE. In Section 5 we present the experimental results of the comparisons outlined in Section 4. Finally, we provide some concluding remarks in Section 6.

\section{OVERVIEW OF T-SNE}

The mathematical foundations of SNE and t-SNE were developed in [2] and [1], respectively; however, to keep the current paper self contained, we review the algorithmic details here.

The basic concept of all data reduction algorithms is to reduce the dimensionality of the data while preserving the features of the high dimensional space. A cost function is used to determine the adequacy of this preservation. This cost can be defined in a number of ways, for SNE this cost is the difference between two probability distributions.

These two distributions (high/low) are created by imposing Gaussian distributions pairwise on the data as shown in equation (1).

$$
p_{i j}=\frac{\exp \left(-\left\|\mathbf{x}_{i}-\mathbf{x}_{j}\right\|^{2} / 2 \sigma^{2}\right)}{\sum_{k \neq l} \exp \left(-\left\|\mathbf{x}_{k}-\mathbf{x}_{l}\right\|^{2} / 2 \sigma^{2}\right)}
$$

where $\mathbf{x}_{i}$ and $\mathbf{x}_{j}$ are two points from the data set and $\sigma$ is selected such that the distribution maintains a fixed perplexity. This perplexity parameter ultimately ensures the Shannon entropy remains fixed consistent between neighbors [2]. One can think of equation (1) as the probability that point $\mathbf{x}_{i}$ would choose point $\mathbf{x}_{j}$ under a neighborhood of points that have a Gaussian distribution.

A similar probability distribution is defined for the low-dimensional space by equation (2) but with a fixed variance so it becomes a function only of the mapped points.

$$
q_{i j}=\frac{\exp \left(-\left\|\mathbf{x}_{i}-\mathbf{x}_{j}\right\|^{2}\right)}{\sum_{k \neq l} \exp \left(-\left\|\mathbf{x}_{k}-\mathbf{x}_{l}\right\|^{2}\right)}
$$

The two probability distributions (1) and (2) are compared by minimizing the Kullback-Liebler divergence (i.e. cost function).

$$
C=K L(P \| Q)=\sum_{i} \sum_{j} p_{i j} \log \left(\frac{p_{i j}}{q_{i j}}\right)
$$

A small value for the cost function would indicate that the two distributions are nearly identical, which means the structure of the low dimensional mapping mirrors that of the high dimension. Equation (3) is typically minimized using standard gradient descent [9]. The function to be minimized (i.e. gradient) is obtained by taking the first derivative of equation (3).

$$
\frac{\delta C}{\delta \mathbf{y}_{i}}=2 \sum_{j}\left(\mathbf{y}_{i}-\mathbf{y}_{j}\right)\left(p_{i j}-q_{i j}+p_{j i}-q_{j i}\right)
$$

In the original SNE paper, the authors describe the "push-pull" nature of the gradient. Essentially, those points which are closer in the high-dimensional space are pushed together and those points which are further apart will be pulled apart. The probabilities between the points act as the force of that push pull property [2].

The differences between SNE and t-SNE are slight. The same probability distribution is used for the high dimensional space, but in the low dimensional space, the Gaussian distribution is replaced 
by a t-distribution with one degree of freedom. Equation (5) shows the low probability mapping for t-SNE.

$$
q_{i j}=\frac{\left(1+\left\|\mathbf{y}_{i}-\mathbf{y}_{j}\right\|^{2}\right)^{-1}}{\sum_{k \neq l}\left(1+\left\|\mathbf{y}_{k}-\mathbf{y}_{l}\right\|^{2}\right)^{-1}}
$$

Using the new low-dimensional probability map, we obtain a slightly different gradient function.

$$
\frac{\delta C}{\delta \mathbf{y}_{i}}=4 \sum_{j}\left(p_{i j}-q_{i j}\right)\left(\mathbf{y}_{i}-\mathbf{y}_{j}\right)\left(1+\left\|\mathbf{y}_{i}-\mathbf{y}_{j}\right\|^{2}\right)^{-1}
$$

Standard gradient descent is typically used to optimize this function. In order to increase the rate of convergence, van der Maaten et al. [1] recommend using supplemental optimization methods such as early exaggeration and adaptive learning rates.

\section{OVERVIEW OF DIMENSIONALITY REDUCTION TECHNIQUES}

In the current work, several existing dimensionality reduction techniques are evaluated for use in dimensionality reduction, prior to using t-SNE for visualization. For completeness, we briefly outline each method used in our experiments.

\subsection{Linear Dimensionality Reduction}

3.1.1 Principal Component Analysis. Principal component analysis is an unsupervised linear dimensionality reduction technique aimed at maximizing the total variance in the data samples. Let $X=\left[\mathbf{x}_{1}, \mathbf{x}_{2}, \ldots, \mathbf{x}_{n}\right] \in \mathbb{R}^{m \times n}$ with $m>>n$, PCA finds an orthonormal basis that maximizes the variance in the column space of $X$. The basis vectors that maximize the variance are the eigenvectors of the sample covariance matrix $C=\frac{1}{n} X X^{T}$ where the data samples in $X$ are mean centered. Once the eigenvectors (i.e. principal components) are computed, dimensionality reduction is performed via the projection $\mathcal{M}=X^{T} U_{k}$ where $U_{k}=\left[\mathbf{u}_{1}, \mathbf{u}_{2}, \ldots, \mathbf{u}_{k}\right]$ is the matrix containing the eigenvectors of $C$ corresponding to the $k$ largest eigenvalues, and $k<<m$ is the dimension of the reduced dimensional space $[7,10,11]$. Once we have the reduced data samples in $\mathcal{M}$, t-SNE is performed on these samples for the purposes of data visualization.

3.1.2 Independent Component Analysis. Independent component analysis is an unsupervised learning technique to find a projection subspace that maximizes the statistical independence of the data, i.e., the value of one component provides no information about the values of any other components. Unlike PCA (which only accounts for first and second order statistics and assumes Gaussian data), ICA attempts to use high-order statistics and maximize the non-Gaussianity of the data [12-15]. The approach proceeds by assuming we have $P$ samples from each class $\mathbf{x}_{1}, \ldots, \mathbf{x}_{P}$ all derived from $P$ independent sources $\mathbf{s}_{1}, \ldots, \mathbf{s}_{P}$. We assume $P$ is the same for both sources and samples in the current outline, however, this is not a requirement. If (as in most classification problems) the number of samples $\mathbf{x}$ in each class is greater than $P$, a PCA pre-processing step is performed to reduce the dimensionality to $P$. We then form a mixing matrix $A$ by

$$
X=A S
$$

where $X=\left[\mathbf{x}_{1}, \mathbf{x}_{2}, \ldots, \mathbf{x}_{P}\right]$ and $S=\left[\mathbf{s}_{1}, \mathbf{s}_{2}, \ldots, \mathbf{s}_{P}\right]$, i.e., the matrix A mixes the sources to reconstruct the samples. The idea is to then determine a matrix (referred to as the un-mixing matrix) $W=A^{-1}$ that best isolates the samples from the sources (i.e., the independent components are contained in the columns of $W$ where $S=W X$ ). In an effort to compute $W$, two common approaches are used, 1) compute $W$ to maximize the mutual information between samples and sources (i.e. maximize the K-L divergence), and 2) compute $W$ to maximize the non-Gaussianity between the samples and sources as measured by either the kurtosis or entropy of the data.

3.1.3 Linear Discriminant Analysis. Linear Discriminant Analysis (LDA) is a supervised, linear dimensionality reduction with the goal of computing a projection matrix $U$ that maximizes the betweenclass means of multi-class data while simultaneously minimizing the within-class means of said data. Such a projection is computed by deriving two scatter matrices to account for variation both within and between different classes. We consider a set of data samples that contain $c$ classes (with associated class labels) ${ }^{1}$. It has been shown that the optimal projection matrix $U$ is the solution to the generalized eigenvalue problem,

$$
S_{B} \mathbf{u}_{p}=\lambda_{p} S_{W} \mathbf{u}_{p}
$$

where $U=\left[\mathbf{u}_{1}, \mathbf{u}_{2}, \ldots, \mathbf{u}_{p}\right]$ corresponds to the $p$ largest eigenvalues $\lambda_{p}, S_{B}$ is a measure of the total scatter between classes, and $S_{W}$ is a measure of the total scatter within each class [11, 16, 17]. Note that there are at most $c-1$ nonzero eigenvalues of (8) therefore the projection space has at most dimension $c-1$. Similar to PCA, dimensionality reduction is performed via the projection $\mathcal{M}=$ $X^{T} U_{p}$ and t-SNE is performed on these samples for the purposes of data visualization.

\subsection{Non-Linear Dimensionality Reduction}

3.2.1 Sammon Mapping. Sammon Mapping is a non-linear reduction technique which derives itself from Classical Scaling. Classical Multi-dimensional Scaling is a linear method which reduces dimensionality using the following cost function:

$$
\phi(Y)=\sum_{i j}\left(d_{i j}^{2}-\left\|\mathbf{y}_{i}-\mathbf{y}_{j}\right\|^{2}\right),
$$

where $\mathrm{y}_{i}$ symbolizes the $i^{\text {th }}$ data point in the low dimensional mapping, and $d_{i j}$ is the Euclidean distance between the high dimensional points $\mathbf{x}_{i}$ and $\mathbf{x}_{j}$.

The downside to this example of multi-dimensional scaling is that large pairwise distances are weighted far heavier than small, which can have a significant effect on the nature of local structures within the data.

Sammon mapping is a variation on this scaling where the cost function has been modified as follows

$$
\phi(Y)=\frac{1}{\sum_{i j} d_{i j}} \sum_{i \neq j} \frac{\left(d_{i j}-\left\|\mathbf{y}_{i}-\mathbf{y}_{j}\right\|\right)^{2}}{d_{i j}} .
$$

${ }^{1} \mathrm{LDA}$ is is the only supervised technique used in this study, and therefore limits itself to labeled data. 
The largest difference between the two costs functions, equations (9) and (10), is the original cost is scaled by the other pairwise distances. By doing so, the cost function more equally weighs the large distances to the small distances which better maintains the local geometry $[5,18]$.

3.2.2 Locally Linear Embedding. Local Linear Embedding (LLE) is an unsupervised, non-linear dimensionality reduction technique which attempts to reduce the data by writing each of the high dimensional points as linear combinations of its $k$ nearest neighbors. These linear combinations are stored as normal vectors in a sparse matrix $W$, where element $w_{i j}$ represents the weight of highdimensional point $\mathbf{x}_{i}$ in the linear combination for high-dimensional point $\mathbf{x}_{i}$. Note that this would be 0 if $\mathbf{x}_{j}$ is not within the neighborhood of $\mathbf{x}_{i}$ (i.e. $d\left(\mathbf{x}_{i}, \mathbf{x}_{j}\right)>k$ ).

By creating these linear combinations, the manifold is approximated linearly as a hyperplane passing through the specific point. Therefore, LLE assesses the error of the low-dimensional, mapped point $\mathrm{y}_{i}$ by computing the magnitude of the Sum Squared Error between the mapped point and a reconstruction of the mapped point using the weights from $W$ and the low-dimensional neighbors. This cost function can be seen in (11).

$$
\phi(Y)=\sum_{i}\left\|\mathbf{y}_{i}-\sum_{j} w_{i j} \mathbf{y}_{j}\right\|^{2}
$$

To avoid the trivial solution that $Y=\mathbf{0}$, this cost function is subject to the constraint that $\sum_{j} w_{i j}=1$. In the seminal paper [18, 19], Roweis and Saul show that the minimum cost is obtained by the smallest non-zero eigenvectors of $(I-W)^{T}(I-W)$ where $I$ is an identity matrix of appropriate size.

\section{EXPERIMENTAL SETUP}

\subsection{Data Set Descriptions}

In keeping with [1], the MNIST and COIL-20 data sets will be used to assess each algorithm. The MNIST data used consists of 2500 instances of the original data set. Each instance is a $28 \times 28$ pixel grayscale image of handwritten digits compiled by high school students and employees of the US Census Bureau [20]. This results in 784-dimensional data with 10 classes, one for each digit. The classes are fairly balanced with the largest class having 280 records and the smallest 222 .

The COIL-20 data set is another collection of grayscale images. The COIL-20 data has larger dimensionality and more classes. Each image is $128 \times 128$ pixels. The entire data set consists of 20 different objects images at 72 different orientations as the object is rotated throughout $360^{\circ}$. This results in a data set containing 1,440 images with a dimensionality of 16,384 [21]. A subset of images taken from both the MNIST data set and the COIL-20 data set are illustrated in Fig. 2 .

\subsection{Assessment Techniques}

While t-SNE has been applied as a general purpose dimensionality reduction tool, this paper will illustrate the findings by observing and evaluating the results with respect to multi-class separability

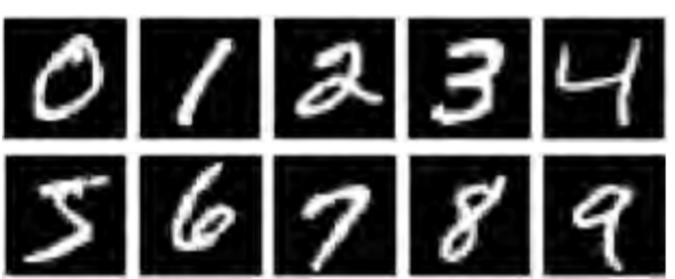

(a)

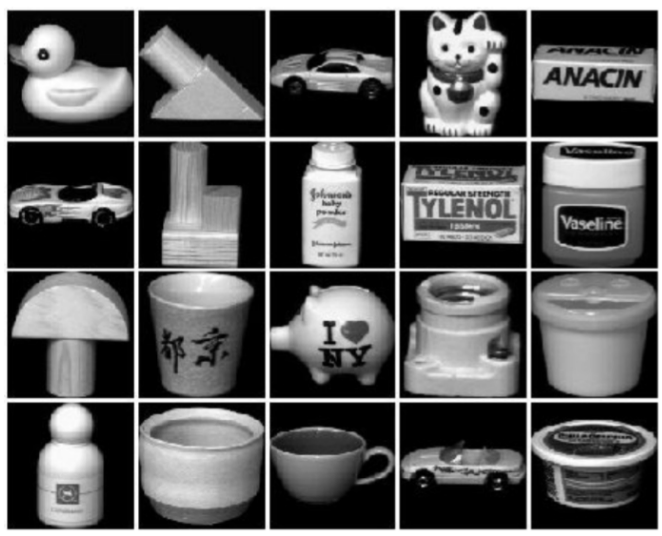

(b)

Figure 2: A subset of sample images from the (a) MNIST data set and (b) COIL-20 data set used for visualization in the current work.

and within-class cohesion. All low-dimension mappings will be in $\mathbb{R}^{2}$. This conforms to the structure of the original t-SNE paper, and also ensures the plots do not suffer from misinterpretation. While each mapping will be discussed in general, one aspect that will be focused on the separation of the classes. The overarching goal of this research is not to find a "best" intermediate dimensionality reduction algorithm, but simply to compare and contrast the selected algorithms. It is likely that one which is the "best" algorithm suited for cluster analysis would not be the "best" algorithm for other purposes.

\subsection{Algorithmic Dimensionality Differences}

As described in Section 3, the intermediate dimensionality reduction algorithms applied in this work are both linear and non-linear as well as supervised and unsupervised. All of the algorithms, with the exception of LDA can reduce the dimensionality to any arbitrary size. In keeping with the original t-SNE paper [1] we will first reduce the data to 30 dimensions prior to performing t-SNE. Because of algorithmic constraints, for LDA the dimensionality will be reducted to $c-1$ dimensions where $c$ is the number of distinct classes $^{2}$.

\footnotetext{
${ }^{2}$ LDA is the only supervised technique used in our experiments and thus would not be an option if the data provided were unlabeled.
} 


\section{EXPERIMENTAL RESULTS}

\subsection{MNIST}

Five different visualizations for the MNIST data can be seen in the left-hand columns of Figures 3 and 4 where the linear dimensionality reduction techniques are illustrated in Fig. 3 and the nonlinear dimensionality reduction techniques are illustrated in Fig. 4. As can be seen from the figures, each intermediate dimensionality reduction technique produces slightly different results. We briefly describe the differences in the plots which result from the different algorithms being used.

PCA (Fig. 3a) shows 10 different clusters. The separation is not perfect (especially dark purple and dark blue) as it appears that PCA has difficulty isolating the numeric digits 2 and 7. This is a result from the fact that in some cases, the handwritten numeric digits appear similar from different users.

For ICA (Fig. 3c), the plot is similar to PCA with the major difference being that the separation between classes using ICA is slightly smaller than using PCA. The smaller between-cluster distance occurs because PCA chooses the components that maximize the variance of the entire data set. If ICA had a greater overall variance then PCA this would not make sense. For ICA, we also see that several of the clusters are split, suggesting class separation of within-class data samples.

LDA (Fig. 3e) shows a much different mapping. Rather than the semi-cohesive clusters seen with PCA and ICA, LDA produced a mapping that has very dense, well-separated clusters. These well defined clusters demonstrate the clear difference in purpose between LDA and PCA. The supervised nature of the algorithm allows it to actively maximize the between-class separation, while simultaneously minimizing the within-class data separation. While LDA's clustering ability is a well known fact, the use of t-SNE to visualize the full LDA space can be a useful tool in exploratory data analysis for labeled data.

Sammon mapping (Fig. 4a) is surprisingly similar to PCA. With Sammon mapping we see 10 distinct clusters, with only 1 cluster being separated, again suggesting class separation of within-class data samples. As with PCA, we also see some scattering of points that do not fit in a cluster. In fact, if the PCA plot were to be reflected across both the $x$ and $y$ axes the two graphs would be near identical.

The final plot (Fig. 4c) is the visualization with LLE as the intermediate data space. This plot shows a close gathering of clusters that, while slightly discernible, are very close together with extensive scattering of data points. In other words, LLE seems to exhibit both poor within-class and between-class separation. In addition, some data clusters are greatly split and form mini-clusters centered around the central mean.

From these plots, we see that using LDA as an intermediate subspace prior to applying t-SNE produces the plot with the largest between-class separation and smallest within-class separation. It's important to observe that each of the intermediate dimensionality reduction techniques produce very different results in the final visualization. This in and of itself suggests that investigating different intermediate data reduction spaces proves to be a useful tool for exploratory data analysis. While van der Maaten et al. [1] use PCA first (Fig 2a), we think it is apparent from our experiments, that the choice of any intermediate dimensionality reduction technique should be based on data set knowledge and desired outcome.

\subsection{COIL-20}

For the COIL-20 data set, the results are even more striking. The resulting mapping can be viewed in the right hand columns of Figures 3 and 4 . In most of the data sets, manifolds (i.e., 1-dimensional curves) can be observed for each of the individual class clusters. These manifolds are rather cyclic in nature, and thus simply speaks to the authenticity of the mappings. For each class, the object is imaged from 72 different orientations throughout $360^{\circ}$. These circular manifolds distinct orientations of each object throughout the entire image sequence.

Using PCA (Fig. 3b), we see that there are two parts to the image. The left side shows a number of manifolds for individual classes. For the most part, these manifolds are well defined, but there are some sections where the manifolds are very close or even on top of each other to some extent. On the right side however, there is a cluster of many overlapping classes. These classes consist mostly of the vehicles, two boxes, and a mug, which can be seen in the bottom in Figure 2.

ICA (Fig. 3d), has similar difficulty distinguishing between some of the classes, however, this appears in the center of the image rather than one side. The other manifolds are decidedly less cylindrical and have better separation overall than the manifolds computed using PCA as the intermediate subspace.

The LDA (Fig. 3f) mapping for the COIL-20 data set is very similar to the mapping we saw with the MINST data. The clusters are of similar size with large separation between classes. The clusters are all similar in size and the distance within the classes is remarkably consistent as well.

With Sammon Mapping (Fig. 4b), the circular manifolds re-emerge and the separation is similar to PCA. Similar to the MNIST data, the Sammon Mapping resembles a mirroring of sorts from the PCA mapping. However, this effect is less profound than we saw with the MNIST data as the problematic classes described above appear in the corner which is different from the observed manifolds.

LLE (Fig. 4d) maps many manifolds in a manner similar to both PCA and Sammon Mapping. The individual manifolds of many of the classes are observed, but the problematic classes are no more cleanly separated. Also, in the center of the image there appear to be a few manifolds which lay atop each other to varying degrees, suggesting difficulty in creating between-class separation.

When these classes are observed, the vehicles and boxes are understandably intermixed as both have a similar, symmetric shape and are about the same size. Similar to the mappings of the MNIST data set, the images in these mappings demonstrate the unique characteristics of the different intermediate data reduction algorithms. 


\subsection{Numerical Analysis}

In order to provide a quantitative comparison, we show that for various cluster algorithms, those clusters which are most defined in Figures 3 and 4 also have the most pronounced numerical values for different cluster metrics.

For comparison, we use purity, which is the proportion in each cluster that come from the majority class, recall, which accounts for the proportion of the class we were able to correctly identify, and silhouette, which measures how similar an object is to its own cluster (within-class scatter) as compared to the other clusters (separation). Numerical results for different clustering metrics can be see in Table 1.

Table 1: Numerical Results (MINST Data)

\begin{tabular}{|l|l|l|l|l|l|l|}
\cline { 2 - 7 } \multicolumn{1}{c|}{} & \multicolumn{6}{c|}{ Clustering Method } \\
\cline { 2 - 7 } \multicolumn{1}{c|}{} & \multicolumn{3}{c|}{ Hierarchical } & \multicolumn{3}{c|}{ Kmeans } \\
\cline { 2 - 7 } & Purity & Recall & Silhouette & Purity & Recall & Silhouette \\
\hline ICA & 0.4652 & 0.4784 & 0.4093 & 0.5 & 0.4819 & 0.4588 \\
\hline PCA & 0.5451 & 0.5694 & 0.4215 & 0.5611 & 0.5444 & 0.4838 \\
\hline LDA & 1 & 1 & 0.8613 & 1 & 1 & 0.8616 \\
\hline SAM & 0.5424 & 0.5694 & 0.4811 & 0.5757 & 0.5535 & 0.5061 \\
\hline LLE & 0.4958 & 0.5 & 0.4149 & 0.4903 & 0.4833 & 0.4511 \\
\hline
\end{tabular}

We see that similar to the visual analysis, LDA significantly outperformed both PCA and ICA. Comparing PCA to ICA, both unsupervised learning methods, PCA was slightly better both in terms of purity and silhouette.

Specifically, purity measures how pure the clusters are in terms of elements from other true classes. If every element of a cluster is a member of the same class, the purity of that cluster would be 1.0. As the table shows, for a given cluster in the ICA mapping, only about two thirds of the data in a cluster falls in the same true class. This would be worrisome as new data classified in this cluster would have almost a $36 \%$ chance of not being in the same true class as the majority of its cluster. As described in the graphical analysis, the lines between the clusters are not clearly defined.

For PCA, that near one-third chance is reduced to about a onefourth chance, but there is still significant room for error. This indicates a larger separation between clusters, but within-class separation (such as can be seen in Fig. 3a) will reduce the purity of that cluster greatly. LDA's purity was the highest with only about a $3.25 \%$ chance that a point classified in a specific cluster would have a different true class than the majority of its cluster. This is also easily seen from the LDA visualizations in Figs. 3e and 3f.

Next we look at the recall metric. Recall looks at each class separately and identifies what proportion of the class were correctly identified. Table 1 shows a distinct separation between LDA and the remaining, unsupervised methods.

ICA had the lowest values with both data sets, followed closely by LLE with neither algorithm breaking 0.5 . As would be expected from the visualizations in Figures 3 and 4, PCA and Sammon Mapping shared nearly identically results with results just above 0.5 for the MNIST set and just below for the COIL-20. LDA on the other had valued a 1.0 on both sets, clearly showing the separation between each of the well-defined clusters found.

Finally, the Silhouette metric is a common metric that combines both the cohesion of the cluster and the separation between clusters. A higher value means that the clusters are more tightly packed and there is ample space between them. Similar to the rest of our analysis, ICA and LLE are the worst of the three algorithms with LDA separating itself in front. The inherent separation between clusters that LDA creates only serves to increase the Silhouette, which is almost twice that of any other algorithm.
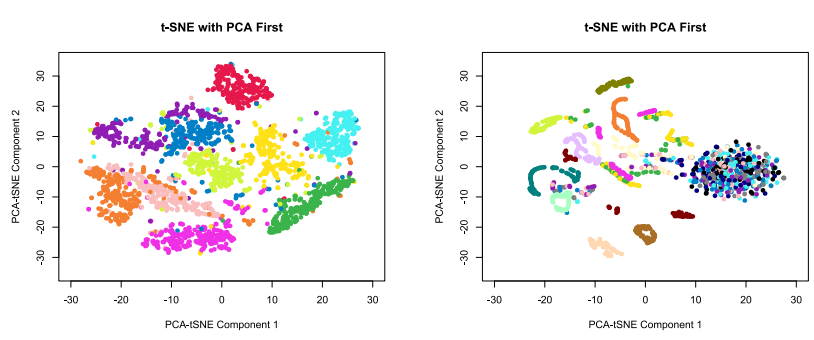

(a) t-SNE visualization of MNIST data (b) t-SNE visualization of COIL20 data with PCA subspace first with PCA subspace first
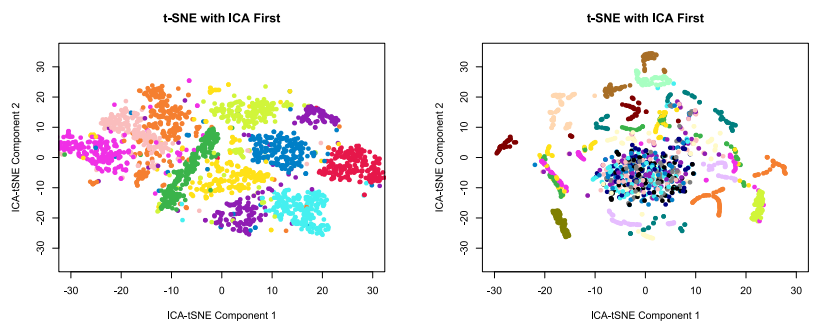

(c) t-SNE visualization of MNIST data (d) t-SNE visualization of COIL20 data with ICA subspace first with ICA subspace first
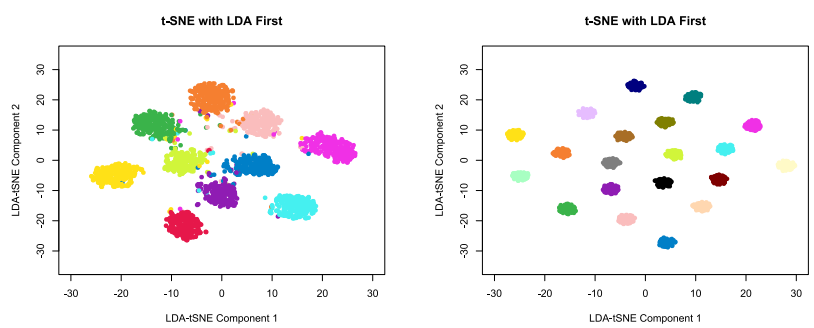

(e) t-SNE visualization of MNIST data (f) t-SNE visualization of COIL20 data with LDA subspace first with LDA subspace first

Figure 3: t-SNE visualizations of the MNIST (left) and COIL20 (right) data for linear intermediate dimensionality reduction techniques PCA (top), ICA (middle) and LDA (bottom).

\section{FINAL REMARKS}

While the focus of this paper required selecting a domain such as clustering so metrics could be compared, the intent is to illustrate 


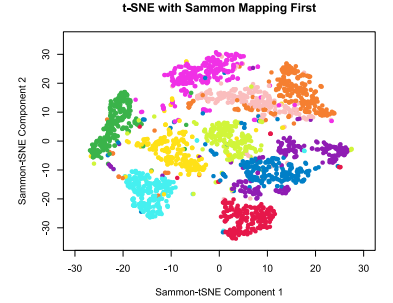

(a) t-SNE visualization of MNIST data with Sammon Mapping subspace first

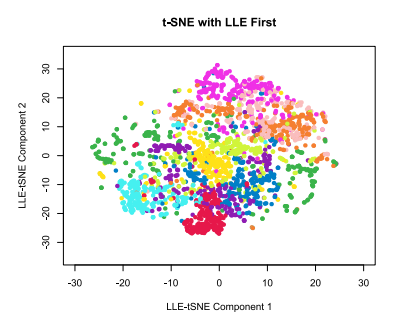

(c) t-SNE visualization of MNIST data with LLE subspace first

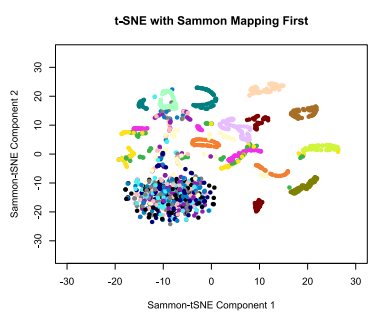

(b) t-SNE visualization of COIL20 data with Sammon Mapping subspace first

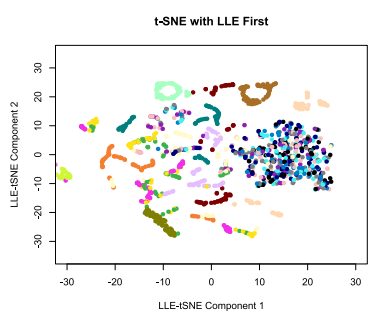

(d) t-SNE visualization of COIL20 data with LLE subspace first

Figure 4: t-SNE visualizations of the MNIST (left) and COIL20 (right) data for the nonlinear intermediate dimensionality reduction techniques Sammon Mapping (top) and LLE (bottom).

the need to consider every step of a data reduction process. t-SNE is not the only dimensionality reduction algorithm which uses intermediate data reduction techniques, and it is important to consider whether the default method for this intermediate reduction is the most useful for a given data and domain.

As the size of data continues to grow, data reduction will only continue to follow in increased importance and usefulness. Being versed in the limitations and advantages of algorithms used to this end allows modern data scientists to extract more meaningful insights this data and sets the stage for greater future developments.

\section{ACKNOWLEDGMENT}

This publication represents research supported by the NAVSEA Warfare Centers NEEC program, grant No. N00174-18-1-0001. Any

opinions, findings, and conclusions or recommendations expressed in this material are those of the author(s) and do not necessarily reflect the views of the Naval Engineering Education Consortium.

\section{REFERENCES}

[1] L. van der Maaten and G. Hinton, "Visualizing data using t-sne," fournal of Machine Learning Research, vol. 9, 2008.

[2] G. E. Hinton and S. T. Roweis, "Stochastic neighbor embedding," in Advances in neural information processing systems, 2003, pp. 857-864.

[3] A. R. Jamieson, M. L. Giger, K. Drukker, H. Li, Y. Yuan, and N. Bhooshan, "Exploring nonlinear feature space dimension reduction and data representation in breast cadx with laplacian eigenmaps and t-sne," Medical Physics, vol. 37, no. 1, pp. 339-351, 2010.

[4] P. Hamel and D. Eck, "Learning features from music audio with deep belief networks," in 11th International Society for Music Information Retrieval Conference (ISMIR 2010)

[5] J. Sammon, "A nonlinear mapping for data structure analysis," IEEE Transactions on Computers, vol. C-18, no. 5, May 1969.

[6] I. Borg and P. J. Groenen, Modern multidimensional scaling: Theory and applications. Springer Science \& Business Media, 2005.

[7] H. Abdi and L. J. Williams, "Principal component analysis," Wiley interdisciplinary reviews: computational statistics, vol. 2, no. 4, pp. 433-459, 2010.

[8] L. Van Der Maaten, "Accelerating t-sne using tree-based algorithms." fournal of machine learning research, vol. 15, no. 1, pp. 3221-3245, 2014.

[9] L. Hasdorff, "Gradient optimization and nonlinear control," 1976.

[10] M. Turk and A. Pentland, "Eigenfaces for recognition," vol. 3, no. 1, pp. 71-86, Mar. 1991.

[11] K. Fukunaga, Introduction to Statistical Pattern Recognition. London, U.K.: Academic, 1990.

[12] A. J. Bell and T. J. Sejnowski, "An information-maximization approach to blind separation and blind deconvolution," Neural Computation, vol. 7, no. 6, p. 1129âĂŞ1159, 1995.

[13] A. Hyvarinen, "Fast and robust fixed-point algorithms for independent component analysis," IEEE Transactions on Neural Networks, vol. 10, no. 3, pp. 626-634, May 1999.

[14] A. HyvãČâĆn̆rinen and E. Oja, "Independent component analysis: algorithms and applications," Neural Networks, vol. 13, no. 4, pp. 411 - 430, 2000.

[15] M. S. Bartlett, J. R. Movellan, and T. J. Sejnowski, "Face recognition by independent component analysis," IEEE Transactions on Neural Networks, vol. 13, no. 6, pp. 1450-1464, Nov 2002.

[16] R. A. Fisher, "The use of multiple measurements in taxonomic problems," Annals of human genetics, vol. 7, no. 2, pp. 179-188, 1936.

[17] P. N. Belhumeur, J. P. Hespanha, and D. J. Kriegman, "Eigenfaces vs. Fisherfaces: Recognition using class specific linear projection," vol. 19, no. 7, pp. 711-720, July 1997.

[18] L. Van Der Maaten, E. Postma, and J. Van den Herik, "Dimensionality reduction: a comparative," 7 Mach Learn Res, vol. 10, pp. 66-71, 2009.

[19] S. T. Roweis and L. K. Saul, "Nonlinear dimensionality reduction by locally linear embedding,” Science, vol. 290, no. 5500, p. 2323âĂȘ2326, 2000.

[20] Y. LeCun, L. Bottou, Y. Bengio, and P. Haffner, "Gradient-based learning applied to document recognition," Proceedings of the IEEE, vol. 86, no. 11, pp. 2278-2324, November 1998.

[21] S. A. Nene, S. K. Nayar, and H. Murase, "Columbia object image library (coil-20)," Technical Report from CUCS, vol. 005, no. 96, February 1996. 